\title{
Current status and future prospect of Super-Kamiokande
}

\author{
Shintaro Ito*i \\ Okayama University, Okayama-city, Okayama, 700-8530, Japan \\ E-mail: s-itodokayama-u.ac.ip
}

\begin{abstract}
Super-Kamiokande (SK) is a water Cherenkov detector with a mass of 50 kton to search for proton decays and measure neutrinos from various sources. The SK experiment has started in 1996, and accumulated data over twenty years. Now we are going to the next stage "Super-KamiokandGadolinium" (SK-Gd) project by loading $0.2 \% \mathrm{Gd}_{2}\left(\mathrm{SO}_{4}\right)_{3} \cdot 8 \mathrm{H}_{2} \mathrm{O}$ into SK to discover supernova relic neutrinos. The current status of the analysis and the future prospect are reported.
\end{abstract}

XIV International Conference on Heavy Quarks and Leptons (HQL2018)

May 27- June 1, 2018

Yamagata Terrsa, Yamagata,Japan

\footnotetext{
* Speaker.

${ }^{\dagger}$ For the Super-Kamiokande Collaboration.
} 


\section{Introduction}

Super-Kamiokande (SK), which is located $1000 \mathrm{~m}$ underground at Ikenoyama, Kamioka, Gifu, Japan, is the world's largest water Cherenkov detector with a mass of 50 kton (Ref. [四]). The main physics targets for the SK experiment are proton decay searches and measuring neutrinos from various sources such as the Sun, the atmosphere, supernova explosions, and artificial neutrino beams. Figure $\square$ shows the schematic of the SK detector. The SK detector is optically separated into two layers, inner detector (ID) and outer detector (OD). The ID is viewed by 11,129 inward-facing 20-inch PMTs and the OD consists of 1,885 eight-inch PMTs used as veto counter for cosmic rays. The fiducial volume of the SK detector for the measurements is two meter from the wall of the ID, which corresponds to 22.5 kton of the ultra-pure water.

The SK experiment is categorized into four different experimental phases denoted as SK-I to SK-IV. SK-I has started in 1996 with 40\% photo coverage. In June 2001, there was an accident that half of PMTs were broken due to the chain reaction of shock wave. Thus, SK-II was carried out with 19\% photo coverage. After SK-II, about 5,000 PMTs were installed to restore the SK detector and SK-III has started in October 2006. In September 2008, new electronics and better water purification system were installed, and SK-IV has been operated until 31 May 2018. The total live time in SK-I to SK-IV corresponds to more than 5,000 days.

Currently we are going to the next experimental phase, the Super-Kamiokande-Gadolinium (SK-Gd) experiment. On 27 June 2015, the SK-Gd project was approved by the SK Collaboration; this is an upgrade of the SK detector, in which $0.2 \%$ gadolinium sulfate octahydrate $\left(\mathrm{Gd}_{2}\left(\mathrm{SO}_{4}\right)_{3} \cdot 8 \mathrm{H}_{2} \mathrm{O}\right)$ is added to the SK water tank (Ref. []]). One of the goals of the SK-Gd experiment is to discover supernova relic neutrinos and to study the star formation history of the universe (Ref. [B] $)$. The current status of the analysis and future prospect of the SK-Gd experiment are reported in this paper.

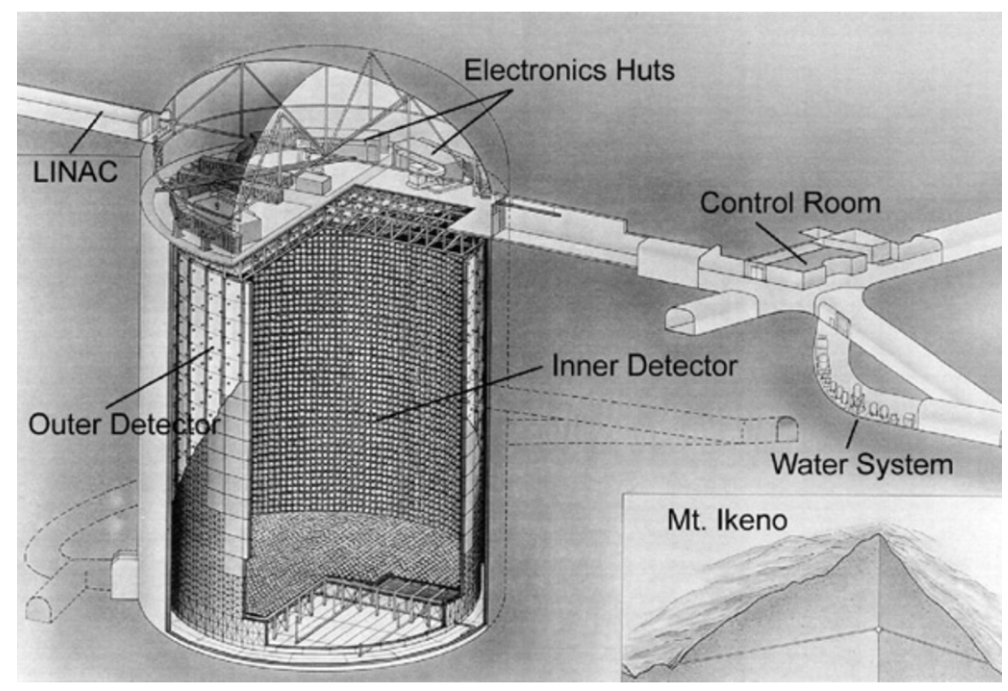

Figure 1: Schematic of the SK detector (Ref. [四). 


\section{Current status of the analysis}

As described in the previous section, measurements of neutrinos from various sources and search for proton decays are performed in the SK experiment over twenty years. The current results of atmospheric and solar neutrinos analysis and proton decay search are briefly presented in this section. See Refs. [4, [1, 6, 团] for more details of these analyses.

Atmospheric neutrinos are one of the main experimentally available neutrino sources, which are generated after the interaction of cosmic rays with the air molecules in atmosphere of Earth, from the decay of secondary particles such as $\pi^{ \pm}$and $K^{ \pm}$mesons. Atmospheric neutrinos can be observed in a wide energy region while the SK detector is sensitive to the energy region from $\mathrm{MeV}$ to $\mathrm{TeV}$ scale. Figure $\square$ shows the result of atmospheric neutrino flux measurement published in Ref. [䧃]. Additionally, updated neutrino oscillation analysis using atmospheric neutrino data was recently published in Ref. [5]. In this analysis, mass hierarchy was studied by comparing upgoing and down-going neutrinos and anti-neutrinos. This result concluded that normal hierarchy is favored and $\delta_{\mathrm{CP}}$ was evaluated to be $\sim 1.33$ if normal hierarchy is assumed. In addition, significance of normal hierarchy is enhanced by combination of SK and T2K data.

For solar neutrino measurement, the SK detector is sensitive to ${ }^{8} \mathrm{~B}$ and hep neutrinos. In 2016, analysis of flux, oscillation, and day/night asymmetry was updated and published in Ref. [6]. Table $\mathbb{W}$ shows the summary of solar neutrino measurements in all experimental period. Because new electronics and better water purification system were installed before SK-IV period, the energy threshold was improved to $3.5 \mathrm{MeV}$. The value of flux is consistent with each experimental period.

In 2017, the result of search for proton decays $p \rightarrow e^{+} \pi^{0}$ and $p \rightarrow \mu^{+} \pi^{0}$ was published in Ref. [प]. At present, candidate event of proton decay was not observed yet in any signal regions. Thus, the upper limits of the lifetime were set for these two decay modes, $>1.6 \times 10^{34}$ years for $p \rightarrow e^{+} \pi^{0}$ and $>7.7 \times 10^{33}$ years for $p \rightarrow \mu^{+} \pi^{0}$.

Table 1: Summary of solar neutrino measurements (preliminary). The errors of the flux respectively represent statistical and systematic uncertainties.

\begin{tabular}{|c||c|c|c|}
\hline Experimental phase & Energy threshold $[\mathrm{MeV}]$ & Livetime [days] & ${ }^{8} \mathrm{~B}$ flux $\left[10^{6} / \mathrm{cm}^{2} / \mathrm{s}\right]$ \\
\hline \hline I & 4.5 & 1496 & $2.38 \pm 0.02 \pm 0.08$ \\
\hline II & 6.5 & 791 & $2.41 \pm 0.05_{-0.15}^{+0.16}$ \\
\hline III & 4.0 & 548 & $2.40 \pm 0.04 \pm 0.05$ \\
\hline IV & 3.5 & 2860 & $2.29 \pm 0.02 \pm 0.04$ \\
\hline
\end{tabular}

\section{Future prospect -SK-Gd-}

\subsection{Introduction}

Supernovae have been occurring since the beginning of the universe, and the neutrinos from all the supernovae have been accumulated as diffuse neutrinos in our universe, called supernova relic neutrinos (SRN). The flux and shape of the energy spectrum highly depend on the star formation so that measurement of SRN would lead us to a better understanding of the star formation history of the universe. 


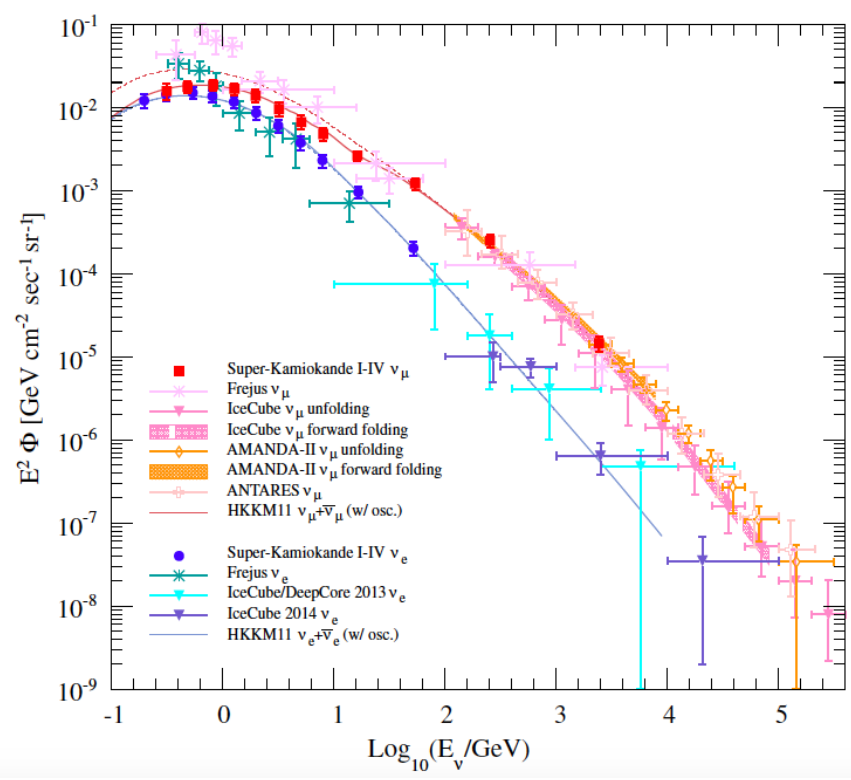

Figure 2: The measured energy spectra of the atmospheric $v_{e}$ (blue curve) and $v_{\mu}$ (red curve) with some other experiments (Ref. [四]). The horizontal axis is the energy of neutrinos and the vertical axis is flux of neutrinos.

Figure [3] shows the expected energy spectra of SRN and backgrounds in SK [D]. Search for SRN signal was performed in the SK experiment, but not observed yet since the signal region is highly limited by atmospheric $\bar{v}_{\mu}$ (Ref. [ [ $\left.\mathrm{Q}, \mathrm{Q}\right]$ ]). In order to reduce background and extend the signal region up to $30 \mathrm{MeV}$, and discover SRN, the SK-Gd project where $0.2 \% \mathrm{Gd}_{2}\left(\mathrm{SO}_{4}\right)_{3} \cdot 8 \mathrm{H}_{2} \mathrm{O}$ is loaded into SK was proposed (Ref. [[]]) and approved by the SK Collaboration.

Gadolinium (Gd) has the largest cross section with neutron so that loading Gd into SK would enables to tag neutron via inverse beta decay $\left(\bar{v}_{e}+p \rightarrow e^{+}+n\right)$. Figure $\mathbb{t}$ shows schematic of neutron tagging by $\mathrm{Gd}$. By adding $0.2 \% \mathrm{Gd}_{2}\left(\mathrm{SO}_{4}\right)_{3} \cdot 8 \mathrm{H}_{2} \mathrm{O}$ (about $0.1 \% \mathrm{Gd}$ ), about $90 \%$ efficiency of neutron capture is expected.

Introduction of neutron tagging into SK also has benefits on other physics studies. For example, atmospheric neutrino analysis described above would be improved because better separation between neutrinos and anti-neutrinos is expected. For solar neutrino analysis and proton decay search, background originating from atmospheric neutrinos or cosmic rays would be reduced. If a galactic supernova occurs, early warning of impending collapse could be detected, approximately a week before explosion. Additionally, pointing accuracy of supernovae would also be improved since supernova $v_{e}$ which has direction information could precisely be separated from $\bar{v}_{e}$.

\subsection{Radio impurities in $\mathrm{Gd}_{2}\left(\mathrm{SO}_{4}\right)_{3} \cdot 8 \mathrm{H}_{2} \mathrm{O}$}

As described in the previous section, the energy threshold was set at about $3.5 \mathrm{MeV}$ to measure low energy solar neutrinos. The concentration of radioactive impurities, ${ }^{226} \mathrm{Ra},{ }^{238} \mathrm{U}$, and ${ }^{232} \mathrm{Th}$ in $\mathrm{Gd}_{2}\left(\mathrm{SO}_{4}\right)_{3} \cdot 8 \mathrm{H}_{2} \mathrm{O}$ loaded water needs to be kept at the same level as in the current SK water in order to continue precise measurements of the solar neutrino spectrum. Therefore, $\mathrm{Gd}_{2}\left(\mathrm{SO}_{4}\right)_{3} \cdot 8 \mathrm{H}_{2} \mathrm{O}$ 


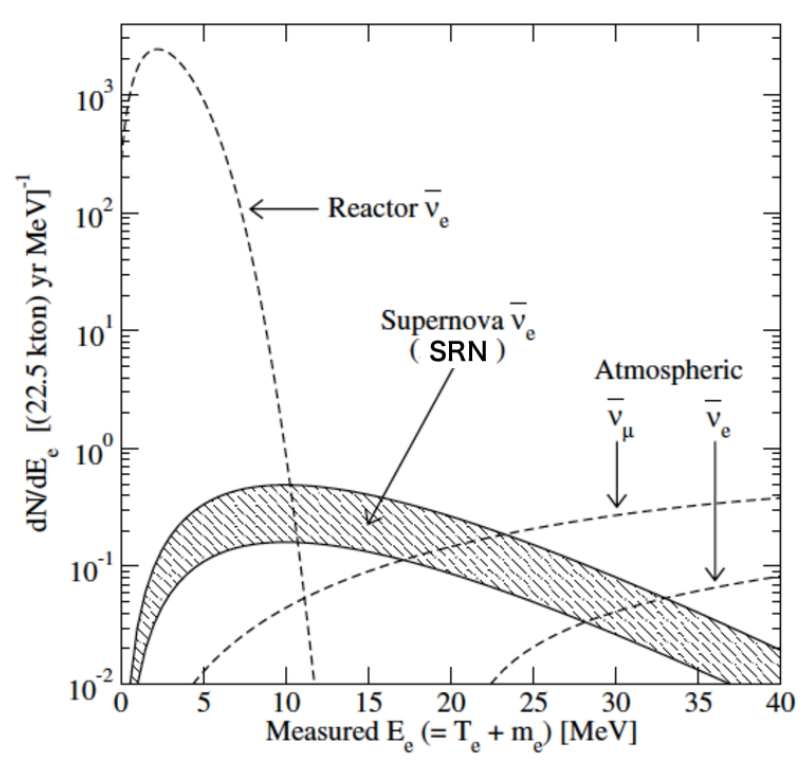

Figure 3: Expected energy spectra of SRN signal and background (Ref. [2]]).

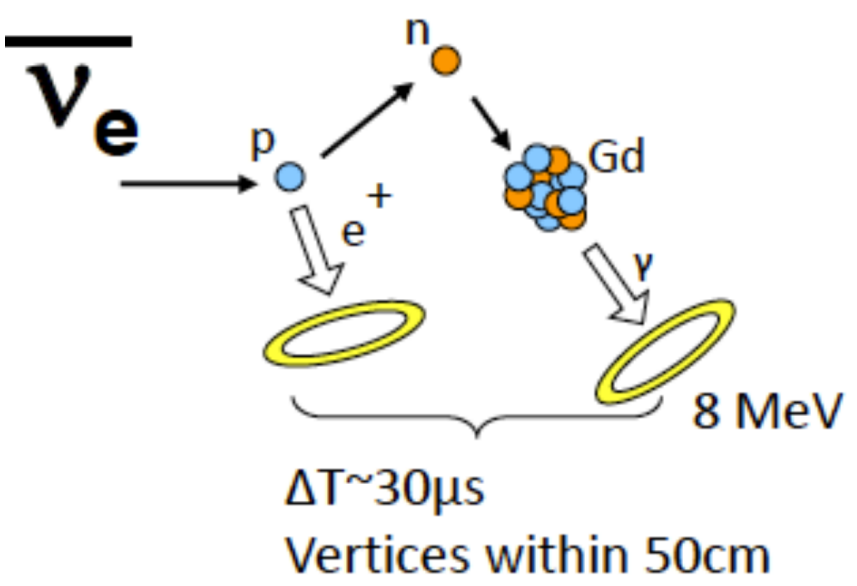

Figure 4: Schematic of neutron tagging by Gd.

should be purified before loading into the SK water tank to avoid interference with the solar neutrino measurements. For the $\mathrm{SK}-\mathrm{Gd}$ experiment, specially purified $\mathrm{Gd}_{2}\left(\mathrm{SO}_{4}\right)_{3} \cdot 8 \mathrm{H}_{2} \mathrm{O}$ samples were produced by several companies, and we developed new methods to precisely measure those radio impurities using ICP-MS (Ref. [ए]]) and germanium detector (Ref. [Ш]). Table $\square$ shows preliminary result of the measurement of radio impurities in $\mathrm{Gd}_{2}\left(\mathrm{SO}_{4}\right)_{3} \cdot 8 \mathrm{H}_{2} \mathrm{O}$ samples. One company finally achieved our requirements of radio impurities.

\subsection{Current status and schedule of SK-Gd}

Before loading $\mathrm{Gd}_{2}\left(\mathrm{SO}_{4}\right)_{3} \cdot 8 \mathrm{H}_{2} \mathrm{O}$ into $\mathrm{SK}$, the water tank should be refurbished to fix the water 
Table 2: Preliminary result of the measurement of radio impurities in $\mathrm{Gd}_{2}\left(\mathrm{SO}_{4}\right)_{3} \cdot 8 \mathrm{H}_{2} \mathrm{O}$ samples. The unit of each radio impurity is $\mathrm{mBq} / \mathrm{kg}$. For comparison between specially purified $\mathrm{Gd}_{2}\left(\mathrm{SO}_{4}\right)_{3} \cdot 8 \mathrm{H}_{2} \mathrm{O}$ and commercially available product, typical values of commercially available product are also listed.

\begin{tabular}{|c||c|c|c|}
\hline Radio impurities & ${ }^{238} \mathrm{U}$ & ${ }^{232} \mathrm{Th}$ & ${ }^{226} \mathrm{Ra}$ \\
\hline \hline Commercial & 50 & 100 & 5 \\
\hline Our goal & $<5$ & $<0.05$ & $<0.5$ \\
\hline \hline Company A & $<0.04$ & $0.02 \pm 0.01$ & $<0.25$ \\
\hline Company B & $<0.04$ & $0.06 \pm 0.01$ & $<0.18$ \\
\hline Company C & $0.64 \pm 0.10$ & $1.77 \pm 0.27$ & $0.70 \pm 0.40$ \\
\hline
\end{tabular}

leakage. On 31 May 2018, the SK experiment was stopped and the tank was opened to fix the water leakage. Drain of water, refurbishment of the tank, replacement of the broken PMTs, and cleaning to remove rust are now in progress. These works will be continued until the end of this September. Then, refilling and circulation of pure water will be performed in October, and normal run using pure water will be carried out in November.

The schedule planning of loading $0.02 \% \mathrm{Gd}_{2}\left(\mathrm{SO}_{4}\right)_{3} \cdot 8 \mathrm{H}_{2} \mathrm{O}$, which corresponds to 10 tons, is ongoing taking into account $\mathrm{T} 2 \mathrm{~K}$ beam availability. The earliest possible $\mathrm{Gd}_{2}\left(\mathrm{SO}_{4}\right)_{3} \cdot 8 \mathrm{H}_{2} \mathrm{O}$ loading into SK would be in late 2019.

After loading $0.02 \% \mathrm{Gd}_{2}\left(\mathrm{SO}_{4}\right)_{3} \cdot 8 \mathrm{H}_{2} \mathrm{O}$, the measurement will be performed about one year, and loading other 90 tons of $\mathrm{Gd}_{2}\left(\mathrm{SO}_{4}\right)_{3} \cdot 8 \mathrm{H}_{2} \mathrm{O}$, and $0.2 \%$ concentrated measurement will be carried out.

\section{Summary}

The SK experiment has been carried out since 1996 to measure neutrinos from various sources and to search for proton decays. The latest results were published in past few years and update of those analysis is in progress. Preparations of the SK-Gd project to discover SRN are also ongoing. We aim to start the SK-Gd experiment in 2019.

\section{Acknowledgments}

This work was supported by the JSPS KAKENHI Grants Grant-in-Aid for Scientific Research on Innovative Areas No. 26104004 and 26104006, Grant-in-Aid for Specially Promoted Research No. 26000003, Grant-in-Aid for Young Scientists No. 17K14290, and Grant-in-Aid for JSPS Research Fellow No. 18J00049. We thank the company who provided the $\mathrm{Gd}_{2}\left(\mathrm{SO}_{4}\right)_{3} \cdot 8 \mathrm{H}_{2} \mathrm{O}$ sample.

\section{References}

[1] S. Fukuda et al., Nucl. Instrum. Methods. A 501, 418 (2003).

[2] J. F. Beacom and M. R. Vagins, Phys. Rev. Lett. 93, 171101 (2004).

[3] S. Horiuchi, J. F. Beacom, and E. Dwek, Phys. Rev. D 79, 083013 (2009).

[4] E. Richard et al., Phys. Rev. D 94, 052001 (2016). 
[5] K. Abe et al., Phys. Rev. D 97, 072001 (2018).

[6] K. Abe et al., Phys. Rev. D 94, 052010 (2016).

[7] K. Abe et al., Phys. Rev. D 95, 012004 (2017).

[8] K. Bays et al., Phys. Rev. D 85, 052007 (2012).

[9] H. Zhang et al., Astropart.Phys. 60 (2015).

[10] S. Ito et al., Prog. Theor. Exp. Phys. 113H01 (2017).

[11] Shintaro Ito, PoS ICRC2017 (available at https://pos.sissa.it/301/1012/pdf). 